\title{
Jellium surface energy beyond the local-density approximation: Self-consistent-field calculations
}

\author{
J. M. Pitarke ${ }^{1}$ and A. G. Eguiluz ${ }^{2}$ \\ ${ }^{1}$ Materia Kondentsatuaren Fisika Saila, Zientzi Fakultatea, Euskal Herriko Unibertsitatea, \\ 644 Posta kutxatila, 48080 Bilbo, Basque Country, Spain \\ and Donostia International Physics Center (DIPC) and Centro Mixto CSIC-UPV/EHU, \\ Donostia, Basque Country, Spain \\ ${ }^{2}$ Department of Physics and Astronomy, The University of Tennessee, Knoxville, TN 37996-1200 \\ and Solid State Division, Oak Ridge National Laboratory, Oak Ridge, TN 37831-6032
}

(November 4, 2018)

\begin{abstract}
We report extensive self-consistent calculations of jellium surface energies, by going beyond the localdensity approximation. The density-response function of a bounded free-electron gas is evaluated within the random-phase approximation, with use of self-consistent electron density profiles. The exchange-correlation energy is then obtained from an exact adiabatic fluctuation-dissipation formula. We also investigate quantum-size effects and the extrapolation of finite-slab calculations to the infinite-width limit.
\end{abstract}

\section{INTRODUCTION}

Density-functional theory (DFT), I in the local-density approximation (LDA) and its various gradient-corrected forms, 2 has been the most widely used method to investigate the properties of solid surfaces. rapidly varying electron density near the surface does not justify a priori the use of local or quasilocal approximations. In particular, these approximations are well known to yield an inaccurate description of the positiondependent exchange-correlation (xc) hole density, they lead to a surface barrier which does not present the correct image-like asymptotic behavior, and there has been a long-standing controversy about the extent to which the LDA accounts for the exact xc surface energy 511 Recently, the wave-function-based Fermi hypernefted-chain (FHNC)12 and quantum Monte Carlo (QMC) 1314 predictions have been found to disagree with modern densityfunctional calculations of jellium surface energies that go beyond a local-density approximation. 1518

In this paper, we present extensive self-consistent calculations of jellium surface energies. The dynamical density-response function of a bounded free electron gas (FEG) is evaluated within the random-phase approximation (RPA) 19 from the knowledge of the non-interacting density-response function. The xc energy of jellium slabs is then obtained via a coupling-constant integration over the interacting density-response function. For comparison, we first consider a simple non-selfconsistent microscopic model of the surface, the so-called infinite-barrier model (IBM), for which analytical insight is possible by virtue of the simple nature of the one-electron wave functions. Full self-consistent calculations are performed by employing either Kohn-Sham or Hartree orbitals to construct the non-interacting density-response function. LDA calculations of jellium surface energies are also carried out, within the same density-response framework, and a comparison between our local and non-local calcu- lations indicate that the error introduced by the LDA is small.

Quantum-size effects (QSE) 20.21 on the surface energy of jellium slabs are also investigated, and the extrapolation of finite-slab calculations to the infinite-width limit is discussed. This issue is important, in view of the potential application of this procedure to the case of more complex slab calculations involving the use of the band structure and/or QMC simulations.

\section{THE JELLIUM SURFACE ENERGY}

We consider a jellium slab of thickness $a$ normal to the $z$ axis, consisting of a fixed uniform positive background of density

$$
n_{+}(z)= \begin{cases}\bar{n}, & -a / 2 \leq z \leq a / 2 \\ 0, & \text { elsewhere }\end{cases}
$$

plus a neutralizing cloud of interacting electrons.

The surface energy is defined as the energy per unit area required to split the solid into two separate halves along a plane. For a solid that is translationally invariant in the plane of the surface, DFT shows thatt

$$
\sigma=\sigma_{s}+\sigma_{e s}+\sigma_{x c},
$$

where $\sigma_{s}$ represents the kinetic surface energy of noninteracting Kohn-Sham electrons

$$
\begin{aligned}
\sigma_{s}= & \frac{m}{4 \pi \hbar^{2}} \sum_{l=1}^{l_{M}}\left(E_{F}^{2}-\varepsilon_{l}^{2}\right)-\frac{\hbar^{2}}{2 m} \frac{q_{F}^{5} a}{10 \pi^{2}} \\
& -\int_{0}^{\infty} d z n(z)\left\{v_{e f f}[n](z)-v_{e f f}[n](0)\right\},
\end{aligned}
$$

$\sigma_{e s}$ is the electrostatic surface energy of all positive and negative charge distributions 


$$
\sigma_{e s}=\frac{1}{2} \int_{0}^{\infty} d z\left[n(z)-n_{+}(z)\right] \varphi(z),
$$

and $\sigma_{x c}$ is the xc surface energy

$$
\sigma_{x c}=\int_{0}^{\infty} d z n(z)\left\{\varepsilon_{x c}[n](z)-\varepsilon_{x c}^{u n i f}(\bar{n})\right\} .
$$

Here, $n(z)$ is the electron density

$$
n(z)=\frac{m}{\pi \hbar^{2}} \sum_{l=1}^{l_{M}}\left(E_{F}-\varepsilon_{l}\right)\left|\phi_{l}(z)\right|^{2},
$$

$v_{\text {eff }}[n](z)$ is the Kohn-Sham effective one-electron potential

$$
v_{e f f}[n](z)=\varphi(z)+v_{x c}[n](z)
$$

$\varphi(z)$ and $v_{x c}[n](z)$ being the electrostatic and xc potential, respectively, $\varepsilon_{x c}[n](z)$ represents an xc energy per particle at point $z$, and $\varepsilon_{x c}^{u n i f}(\bar{n})$ is the xc energy per particle of a uniform electron gas of density $\bar{n} . \varepsilon_{l}$ and $\phi_{l}(z)$ are eigenvalues and eigenfunctions of the one-dimensional Kohn-Sham hamiltonian describing motion normal to the surface, $l_{M}$ denotes the highest occupied level, $E_{F}$ represents the Fermi level, and $q_{F}=\left(3 \pi^{2} \bar{n}\right)^{1 / 3}$ is the Fermi momentum. In the LDA, the xc surface energy is obtained by simply replacing $\varepsilon_{x c}[n](z)$ by the xc energy per particle of a uniform electron gas of density $n(z)$, i.e.,

$$
\sigma_{x c}^{L D A}=\int_{0}^{\infty} d z n(z)\left\{\varepsilon_{x c}^{u n i f}[n(z)]-\varepsilon_{x c}^{u n i f}(\bar{n})\right\} .
$$

The exact xc energy per particle at point $\mathbf{r}$ can be obtained as the energy due to the interaction between an electron at $\mathbf{r}$ and its averaged xc hole:

$$
\varepsilon_{x c}[n](\mathbf{r})=\frac{e^{2}}{2} \int d \mathbf{r}^{\prime} \frac{\bar{n}_{x c}\left(\mathbf{r}, \mathbf{r}^{\prime}\right)}{\left|\mathbf{r}-\mathbf{r}^{\prime}\right|}
$$

where $\bar{n}_{x c}\left(\mathbf{r}, \mathbf{r}^{\prime}\right)$ is defined by adiabatically switching on the electron-electron interaction via a coupling constant $\lambda$, i.e., $v^{\lambda}\left(\mathbf{r}-\mathbf{r}^{\prime}\right)=\lambda e^{2} /\left|\mathbf{r}-\mathbf{r}^{\prime}\right|$ and by adding, at the same time, an external potential so as to maintain the true $(\lambda=1)$ ground-state density in the presence of the modified electron-electron interaction. One writes 9

$$
\bar{n}_{x c}\left(\mathbf{r}, \mathbf{r}^{\prime}\right)=\int_{0}^{1} d \lambda n_{x c}^{\lambda}\left(\mathbf{r}, \mathbf{r}^{\prime}\right)
$$

where $n_{x c}^{\lambda}\left(\mathbf{r}, \mathbf{r}^{\prime}\right)$ is the xc-hole density of a fictitious system at coupling strength $\lambda$. Using the fluctuationdissipation theorem, 22 one may write

$$
\begin{aligned}
n_{x c}^{\lambda}\left(\mathbf{r}, \mathbf{r}^{\prime}\right)=\frac{1}{n(\mathbf{r})}[ & -\frac{\hbar}{\pi} \int_{0}^{\infty} d \omega \chi^{\lambda}\left(\mathbf{r}, \mathbf{r}^{\prime} ; i \omega\right) \\
& \left.-n(\mathbf{r}) \delta\left(\mathbf{r}-\mathbf{r}^{\prime}\right)\right]
\end{aligned}
$$

where $\chi^{\lambda}\left(\mathbf{r}, \mathbf{r}^{\prime} ; \omega\right)$ represents the so-called densityresponse function of the electron system.
Time-dependent DFT (TDDFT) shows that the exact density-response function satisfies the integral equation 23

$$
\begin{aligned}
& \chi^{\lambda}\left(\mathbf{r}, \mathbf{r}^{\prime} ; \omega\right)=\chi^{0}\left(\mathbf{r}, \mathbf{r}^{\prime} ; \omega\right)+\int d \mathbf{r}_{1} \int \mathrm{d} \mathbf{r}_{2} \\
& \quad \times \chi^{0}\left(\mathbf{r}, \mathbf{r}_{1} ; \omega\right)\left\{v^{\lambda}\left(\mathbf{r}_{1}, \mathbf{r}_{2}\right)+f_{x c}^{\lambda}[n]\left(\mathbf{r}_{1}, \mathbf{r}_{2} ; \omega\right)\right\} \\
& \quad \times \chi^{\lambda}\left(\mathbf{r}_{2}, \mathbf{r}^{\prime} ; \omega\right),
\end{aligned}
$$

where

$$
f_{x c}^{\lambda}[n]\left(\mathbf{r}, \mathbf{r}^{\prime} ; \omega\right)=\frac{\delta v_{x c}^{\lambda}[n](\mathbf{r}, \omega)}{\delta n\left(\mathbf{r}^{\prime}, \omega\right)}
$$

$v_{x c}^{\lambda}[n](\mathbf{r}, \omega)$ being the exact frequency-dependent $\mathrm{xc}$ potential, 24 and $\chi^{0}\left(\mathbf{r}, \mathbf{r}^{\prime} ; \omega\right)$, the density-response function of non-interacting Kohn-Sham electrons, i.e., independent electrons moving in the effective potential $v_{\text {eff }}[n](\mathbf{r})$ entering the Kohn-Sham equation of DFT.

If the interacting density-response function $\chi^{\lambda}\left(\mathbf{r}, \mathbf{r}^{\prime} ; \omega\right)$ is replaced by $\chi^{0}\left(\mathbf{r}, \mathbf{r}^{\prime} ; \omega\right)$, Eq. (9) yields the exact exchange energy per particle at point $\mathbf{r}, \varepsilon_{x}[n](\mathbf{r})$. The RPA $\varepsilon_{x c}[n](\mathbf{r})$ is derived by simply setting the xc kernel of Eq. (13) equal to zero.25 In the so-called time-dependent local-density approximation (TDLDA)26 or, equivalently, adiabatic local-density approximation (ALDA), the exact long-wavelength limit of the static $(\omega=0)$ xc kernel is used, i.e.,

$$
f_{x c}^{\lambda, A L D A}[n]\left(\mathbf{r}, \mathbf{r}^{\prime} ; \omega\right)=\frac{d v_{x c}^{\lambda, \text { unif }}[n(\mathbf{r})]}{d n(\mathbf{r})} \delta\left(\mathbf{r}-\mathbf{r}^{\prime}\right),
$$

$v_{x c}^{\lambda, u n i f}[n(\mathbf{r})]$ being the xc potential of a uniform electron gas of density $n(\mathbf{r})$.

For a uniform electron gas of density $n$, introduction of Eq. (11) into Eq. (10) and then Eq. (10) into Eq. (9) yields

$$
\begin{aligned}
\varepsilon_{x c}^{u n i f}(n)= & \frac{1}{2} \int \frac{d \mathbf{q}}{(2 \pi)^{3}} v(q) \\
& \times\left[-\frac{\hbar}{\pi n} \int_{0}^{1} d \lambda \int_{0}^{\infty} d \omega \chi^{\lambda}(q, i \omega)-1\right],
\end{aligned}
$$

where $v(q)$ and $\chi^{\lambda}(q, \omega)$ represent three-dimensional Fourier transforms of the Coulomb potential and the density-response function, respectively. If the interacting density-response function $\chi^{\lambda}(q, \omega)$ is replaced by the Lindhard function $\chi^{0}(q, \omega), 27$ Eq. (15) yields the exact exchange energy per particle of a uniform electron gas,

$$
\varepsilon_{x}^{u n i f}(n)=-\frac{3 e^{2}}{4 \pi}\left(3 \pi^{2} n\right)^{1 / 3},
$$

and the LDA exchange surface energy

$$
\sigma_{x}^{L D A}=\frac{3 e^{2}}{8 \pi} \bar{n} \int_{0}^{\infty} d z^{\prime} \rho\left(z^{\prime}\right)\left[1-\rho^{1 / 3}\left(z^{\prime}\right)\right],
$$

where $\rho(z)=n(z) / \bar{n}$ represents the normalized electron density, and $z^{\prime}=2 q_{F} z$. 
For a jellium slab, which is translationally invariant only in a plane perpendicular to the $z$ axis, one finds

$$
\begin{gathered}
\varepsilon_{x c}[n](z)=\frac{1}{2} \int \frac{d \mathbf{q}_{\|}}{(2 \pi)^{2}} \int d z^{\prime} v\left(z, z^{\prime} ; q_{\|}\right)\left[-\frac{\hbar}{\pi n(z)}\right. \\
\left.\times \int_{0}^{1} d \lambda \int_{0}^{\infty} d \omega \chi^{\lambda}\left(z, z^{\prime} ; q_{\|}, i \omega\right)-\delta\left(z-z^{\prime}\right)\right],
\end{gathered}
$$

where $v\left(z, z^{\prime} ; q_{\|}\right)$and $\chi^{\lambda}\left(z, z^{\prime} ; q_{\|}, \omega\right)$ represent twodimensional Fourier transforms of the Coulomb potential and the density-response function, and $\mathbf{q}_{\|}$is a wave vector parallel to the surface.

To find $\chi^{\lambda}\left(z, z^{\prime} ; q_{\|}, \omega\right)$, we follow the method described in Ref. 28. We first assume that $g(z)$ vanishes at a distance $z_{0}$ from either jellium edge 29 and expand the wave functions $\phi_{l}(z)$ in a Fourier sine series. We then introduce a double-cosine Fourier representation for the densityresponse function, which allows us to transform Eq. (12) into a matrix equation that is solved numerically. The integrals of Eqs. (5) and (18) over the coordinate normal to the surface can be performed analytically, and we find an explicit expression for the xc surface energy, in terms of the eigenvalues $\varepsilon_{l}$ and the Fourier coefficients of the eigenfunctions $\phi_{l}(z)$ (see Appendix A).

\section{THE INFINITE BARRIER MODEL}

In this section we consider the simplest possible microscopic model of the jellium slab, namely the so-called infinite barrier model (IBM).30 This non-selfconsistent model has been widely discussed, 31 B3 because of the simplicity of the one-electron wave functions. Nevertheless, we are not aware of any theoretical description of the extrapolation, within this model, of finite-slab calculations to the infinite-width limit. Since this issue may be important, in view of its potential application to the case of more complex self-consistent calculations, we present below a detailed analysis of QSE on the surface energy of an electron system confined in an infinite well.

In the IBM, the Kohn-Sham effective one-electron potential $v_{e f f}[n](z)$ is replaced by infinitely high potential walls at a distance $z_{0}$ outside the jellium slab,

$$
v_{\text {eff }}(z)= \begin{cases}0, & -a / 2-z_{0} \leq a / 2+z_{0} \\ \infty, & \text { elsewhere }\end{cases}
$$

with $z_{0}$ chosen so as to ensure charge neutrality, i.e.,

$$
\rho_{0}\left(a+2 z_{0}\right) \equiv 2 \int_{0}^{a / 2+z_{0}} d z \rho(z)=a,
$$

$\rho_{0}$ representing the normalized electron density averaged over the region between the two infinite barriers.

Hence, the normalized Kohn-Sham one-electron wave functions and energies are

$$
\phi_{l}(z)=\sqrt{\frac{2}{d}} \sin \left(\frac{l \pi}{d} z\right)
$$

and

$$
\varepsilon_{l}=\frac{\hbar^{2}}{2 m}\left(\frac{l \pi}{d}\right)^{2},
$$

with $d=a+2 z_{0}$. Introduction of these eigenfunctions and eigenvalues into Eq. (6) yields the IBM electron density, and no attempt is then made to alter $v_{e f f}[n](z)$ using this computed density.

In the infinite-width (iw) dimit and for $z>0$, Eq. (6) yields the well-known resulta

$$
[\rho(z)]_{i w}=\left[1+3 \tilde{z}^{-3}(\tilde{z} \cos \tilde{z}-\sin \tilde{z})\right] \Theta(-\tilde{z}),
$$

where $\tilde{z}=2 q_{F}(z-d / 2)$. By carrying out the integration of Eq. 200 one then easily finds

$$
\left[\rho_{0}\right]_{i w}=1-\frac{3}{4 x}
$$

and

$$
\left[z_{0}\right]_{i w}=(3 / 16) \lambda_{F},
$$

where the dimensionless parameter $x=2 d / \lambda_{F}$ has been introduced, $\lambda_{F}=2 \pi / q_{F}$ being the Fermi wavelength.

Instead, for a finite slab one finds

$$
\rho_{0}=\frac{3}{2 x} \sum_{l=1}^{l_{M}}\left[1-\left(\frac{l}{x}\right)^{2}\right]
$$

and

$$
z_{0}=\lambda_{F} \frac{x}{4}\left\{1-\frac{3}{2 x} \sum_{l=1}^{l_{M}}\left[1-\left(\frac{l}{x}\right)^{2}\right]\right\},
$$

with the highest occupied level $l_{M}$ being the largest integer less than or equal to $x$.

The dependence of the average electron density $\rho_{0}$ on the parameter $x$, as obtained from Eq. (26), is shown in Fig. 1. This figure clearly shows the oscillatory character of this quantity, which is a consequence of the so-called QSE first reported by Schulte. 20 As $d$ increases, new subbands for the $z$ motion become occupied. One easily sees that such a new subband falls below the Fermi level every time $d$ is a multiple of $\lambda_{F} / 2$, i.e., every time $x$ is integer. When a new subband is pulled below the Fermi level, the parallel Fermi sea built upon the newly occupied subband acquires more electrons, thereby increasing $\rho_{0}$. However, this effect is eventually overcome by the fact that all the subbands for the $z$ motion get deeper with increasing film thickness. When $x$ has increased by unity, a new subband begins to be filled and a new oscillation begins.

We define the threshold value $\left[\rho_{0}\right]_{1}$ of the normalized average electron density $\rho_{0}$ corresponding to a jellium 
slab with $x$ integer. By using the Euler-Maclaurin summation formula 34 Eq. (26) with $x=n$ yields a local minimum,

$$
\left[\rho_{0}\right]_{1}=1-\frac{3}{4 x}\left(1+\frac{1}{3 x}\right) .
$$

We also define $\left[\rho_{0}\right]_{2}$ corresponding to a jellium slab with $x=n+1 / 2$, and find

$$
\left[\rho_{0}\right]_{2}=1-\frac{3}{4 x}\left(1-\frac{1}{6 x}\right) .
$$

As $x$ increases, both Eqs. (28) and (29) approach the infinite-width asymptotic behavior of Eq. (24). Although $\left[\rho_{0}\right]_{1}$ and $\left[\rho_{0}\right]_{2}$ represent the actual value of $\rho_{0}$ only at $x=$ $n$ and $x=n+1 / 2$, respectively, they are exhibited in Fig. 1 for all values of $x$. This figure shows that Eqs. (28) and (29) represent enveloping lines of the actual oscillating behavior of $\rho_{0}$. Hence, the amplitude of the oscillations is

$$
\left[\rho_{0}\right]_{2}-\left[\rho_{0}\right]_{1}=\frac{1}{2 x},
$$

thus decaying linearly with the thickness of the slab. Moreover, the infinite-width limit of Eq. (24) is obtained from the enveloping lines of Eqs. (28) and (29), as follows

$$
\left[\rho_{0}\right]_{i w}=\frac{\left[\rho_{0}\right]_{1}+2\left[\rho_{0}\right]_{2}}{3} .
$$

Fig. 2 shows that a similar oscillatory behavior is exhibited by the normalized electron density $\rho(z)$ at the center of the slab $(z=0)$ :

$$
\rho(0)=\frac{3}{x} \sum_{l=1}^{l_{M}^{\prime}}\left[1-\left(\frac{2 l-1}{x}\right)^{2}\right],
$$

where $l_{M}^{\prime}$ is the largest integer less than or equal to $(x+$ 1)/2. Also shown in Fig. 2 are the enveloping lines

$$
[\rho(0)]_{1}=1-\frac{1}{x^{2}}
$$

and

$$
[\rho(0)]_{2}=1+\frac{1}{2 x^{2}}
$$

which represent the actual value of $\rho(0)$ for $x$ odd and even, respectively. The infinite-width limit $[\rho(0)]_{i w}=1$ is obtained as in Eq. (31), with $\rho(0)$ instead of $\rho_{0}$.

At the threshold width for which the $n$th subband for the $z$ motion is first occupied $(x=n)$, the normalized average electron density $\rho_{0}$ is smaller than in the infinitewidth limit, i.e., $\left[\rho_{0}\right]_{1}<\left[\rho_{0}\right]_{i w}$, which requires that the positive background be cut-off further from the infinite wells, i.e., $z_{0}$ is a local maximum. This is illustrated in Fig. 3, where the dependence of the ratio $z_{0} /\left[z_{0}\right]_{i w}$ on the parameter $x$ is exhibited, as obtained from Eq. 27). Also plotted in this figure are the enveloping lines

$$
\left[z_{0}\right]_{1}=\left[z_{0}\right]_{i w}\left[1+\frac{1}{3 x}\right]
$$

and

$$
\left[z_{0}\right]_{2}=\left[z_{0}\right]_{i w}\left[1-\frac{1}{6 x}\right],
$$

which coincide with the actual value of $z_{0}$ for $x$ integer $(x=n)$ and for $x=n+1 / 2$, respectively. An inspection of Eqs. (35) and (36) easily shows that the infinite-width limit of Eq. (25) is obtained as in Eq. (31), with $\rho_{0}$ replaced by $z_{0}$.

\section{A. Kinetic surface energy}

An explicit expression for the surface-energy term $\sigma_{s}$ is obtained by substituting Eqs. (19) and (22) into Eq. (3). One finds

$$
\begin{aligned}
\sigma_{s}=\left[\sigma_{s}\right]_{i w} & \left\{10 \sum_{l=1}^{l_{M}}\left[1-\left(\frac{l}{x}\right)^{2}\right]\right. \\
& \left.-12 \sum_{l=1}^{l_{M}}\left[1-\left(\frac{l}{x}\right)^{4}\right]\right\},
\end{aligned}
$$

where

$$
\left[\sigma_{s}\right]_{i w}=\frac{\hbar^{2}}{2 m} \frac{q_{F}^{4}}{80 \pi}
$$

is the often-derived value of the IBM surface-energy term $\sigma_{s}$ of a semi-infinite medium. 1 For $x$ integer, the EulerMaclaurin summation formula now yields

$$
\left[\sigma_{s}\right]_{1}=\left[\sigma_{s}\right]_{i w}\left[1-\frac{1}{3 x}\left(4-\frac{1}{x^{2}}\right)\right],
$$

while for $x=n+1 / 2$ one finds

$$
\left[\sigma_{s}\right]_{2}=\left[\sigma_{s}\right]_{i w}\left[1+\frac{1}{6 x}\left(4-\frac{1}{x^{2}}\right)-\frac{1}{8 x^{3}}\right] .
$$

The dependence of the ratio $\sigma_{s} /\left[\sigma_{s}\right]_{i w}$ on the parameter $x$, as obtained from Eq. (37), is exhibited in Fig. 4. Also plotted in this figure are $\left[\sigma_{s}\right]_{1}$ and $\left[\sigma_{s}\right]_{2}$. While $\left[\sigma_{s}\right]_{1}$ represents the actual surface-energy term $\sigma_{s}$ at the local minima $(x=n)$, local maxima slightly deviate from $x=n+1 / 2$, especially for the smallest values of $x$. For large values of $x$, Eq. (40) approaches the upper enveloping line of the true oscillating behavior of $\sigma_{s}$. Furthermore, one finds

$$
\left[\sigma_{s}\right]_{i w}=\frac{\left[\sigma_{s}\right]_{1}+2\left[\sigma_{s}\right]_{2}}{3}+O\left(x^{-3}\right),
$$

where the first term quickly approaches the actual infinite-width limit of Eq. (38), as shown in Fig. 4. 


\section{B. Exchange surface energy}

The LDA exchange surface energy is obtained by simply introducing the normalized electron density into Eq. (17). In the infinite-width limit $\rho(z)$ is that of Eq. 23 . and numerical integration leads to the following result 5

$$
\left[\sigma_{x}^{L D A}\right]_{i w}=6.3179 \times 10^{-3} \frac{e^{2}}{r_{s}^{3}},
$$

as reported in Ref. 5 .

For the actual IBM electron density of a finite slab of thickness $d=x \lambda_{F} / 2$, introduction of Eq. (6) into Eq. (17) yields the ratio $\sigma_{x}^{L D A} /\left[\sigma_{x}^{L D A}\right]_{i w}$ shown in Fig. 5, as a function of $x$. As in the case of the kinetic surface energy, we observe a considerable quantum-size effect, even for slab widths as large as five times the Fermi wavelength. The amplitude of the oscillations is also found to decay linearly with the thickness of the slab. However, explicit expressions for the enveloping lines $\left[\sigma_{x}^{L D A}\right]_{1}$ and $\left[\sigma_{x}^{L D A}\right]_{2}$ are not available; hence, for each integer value of $x(x=n)$ we take $\left[\sigma_{x}^{L D A}\right]_{1}=\sigma_{x}^{L D A}(n)$ and approximate $\left[\sigma_{x}^{L D A}\right]_{2}$ as follows

$$
\left[\sigma_{x}^{L D A}\right]_{2} \sim \frac{\left[\sigma_{x}^{L D A}\right]_{2}^{-}+\left[\sigma_{x}^{L D A}\right]_{2}^{+}}{2}
$$

where $\left[\sigma_{x}^{L D A}\right]_{2}^{-}=\sigma_{x}^{L D A}(n-1 / 2)$ and $\left[\sigma_{x}^{L D A}\right]_{2}^{+}=$ $\sigma_{x}^{L D A}(n+1 / 2)$. Assuming that the infinite-width limit can be extrapolated as in Eq. (41), we use the relation

$$
[\sigma]_{i w} \sim \frac{[\sigma]_{2}^{-}+[\sigma]_{1}+[\sigma]_{2}^{+}}{3},
$$

with $\sigma$ replaced by $\sigma_{x}^{L D A}$.

This procedure yields the results represented in Fig. 5 by open circles, which quickly approach the actual infinite-width limit of Eq. (42). The normalized LDA exchange surface energies obtained from Eq. (44) are also shown in Fig. 6 (open circles), but now for values of $x$ up to 100 and together with local maxima and minima, $\left[\sigma_{x}^{L D A}\right]_{1} /\left[\sigma_{x}^{L D A}\right]_{i w}$ and $\left[\sigma_{x}^{L D A}\right]_{2}^{ \pm} /\left[\sigma_{x}^{L D A}\right]_{i w}$, respectively (dashed lines). While the amplitude of the quantum-size effect amounts, for slab widths as large as 50 times the Fermi wavelength $(x=100)$, to $1 \%$ of the actual infinite-width limit, for slab widths of only 5 times the Fermi wavelength $(x=10)$ the numerical error introduced by the use of Eq. (44) is found to be within $0.02 \%$.

Within the IBM, we have also calculated exact exchange surface energies by introducing Eqs. (15) and (18) into Eq. (5) with $\chi^{0}(q, \omega)$ and $\chi^{0}\left(z, z^{\prime} ; q_{\|}, \omega\right)$ instead of $\chi^{\lambda}(q, \omega)$ and $\chi^{\lambda}\left(z, z^{\prime} ; q_{\|}, \omega\right)$. We have found an oscillatory behavior of this exact contribution to the surface energy that is similar to that shown in Fig. 5, and have tested the approximation of Eq. (44) (with $\sigma$ replaced by $\sigma_{x}$ ) for slab widths as large as 100 times the Fermi wavelength $(x=200)$. We have found

$$
\left[\sigma_{x}\right]_{i w}=4.0700 \times 10^{-3} \frac{e^{2}}{r_{s}^{3}},
$$

which is in exactagreement with the result obtained by Harris and Jones5 for the semi-infinite medium.

The extrapolated exact exchange surface energies, as obtained from Eq. (44) with $\sigma$ replaced by $\sigma_{x}$, are represented in Fig. 6 versus $x$ (only integer values of $x$ have been considered) by open squares, showing that it approaches the infinite-width limit of Eq. (45). Although the extrapolation procedure dictated by Eq. (44) does not converge as rapidly for $\sigma_{x}$ as for $\sigma_{x}^{L D A}$, the numerical error introduced by the use of this procedure to evaluate $\sigma_{x}$ is still found to be within $0.7 \%$ for slab widths of only 5 times the Fermi wavelength $(x=10)$.

\section{Exchange-correlation surface energy}

For a given electron density, the LDA xc surface energy is obtained by introducing into Eq. (8) the xc energy per particle of a uniform electron gas, $\varepsilon_{x c}^{u n i f}$, which we have calculated with use of either the Wigner intermolation formula 36 the Perdew-Zunger parametrization 37 of the QMC calculation of Ceperley and Alder, 38 or Eq. (15) with the RPA interacting density-response function. We have found quantum-size effects and the extrapolation of finite-slab calculations to the infinite-width limit to be similar to those exhibited by kinetic and exchange contributions to the surface energy. Hence, for each value of $r_{s}$ and a given number $n$ of occupied subbands, we have considered three different values of the parameter $x: n-1 / 2$, $n$, and $n+1 / 2$, and have used Eq. (44) with $\sigma$ replaced by $\sigma_{x c}^{L D A}$. When either the Wigner interpolation formula or the RPA are used for $\varepsilon_{x c}^{u n i f}$, the extrapolation procedure dictated by Eq. (44) yields LDA xc surface energies that are in agreement with those reported for a semi-infinite medium in Refs. 6 and 9, respectively. Since the Wigner correlation energy changes very little with the electron density, as compared with RPA or Perdew-Zunger correlation energies, it yields LDA xc surface energies that are too small.

For each $r_{s}$ and given values of $x$, we have also computed non-local xc surface energies from Eq. (5), as obtained with the xc energy of Eqs. (15) and (18) computed in the RPA. We have found that the extrapolation of these finite-slab calculations to the infinitewidth limit is accurately described by Eq. (44), but now with $\sigma$ replaced by $\sigma_{x c}$. Table I shows our extrapolated RPA correlation-only and exchange-correlation surface energies, $\sigma_{c}$ and $\sigma_{x c}$, together with the RPAbased LPA surface energies, $\sigma_{c}^{L D A}$ and $\sigma_{x c}^{L D A}$, described above.39.40 While the local-density approximation overestimates, within the IBM, the exact exchange surface energy by $55 \%$ [see Eqs. (42) and (45)] for all values of $r_{s}$, it largely underestimates the RPA correlation surface energy by $78 \%$ at $r_{s}=2$ and $64 \%$ at $r_{s}=6$. Large and opposite separate corrections to the LDA for exchange 
and correlation nearly compensate, as discussed in Refs. 6 and 9, and yield LDA xc surface energies that are, over the entire metallic density range, within about $6.5 \%$ of their non-local counterparts.

For comparison, Table I also shows the extrapolated LDA correlation-only and exchange-correlation surface energies, $\sigma_{c}^{L D A^{\prime}}$ and $\sigma_{x c}^{L D A^{\prime}}$, that we have obtained with use of the Perdew-Zunger parametrization for $\varepsilon_{x c}^{u n i f}$. Although RPA and Perdew-Zunger correlation energies of a uniform electron gas differ, for $r_{s} \sim 0$-10, by $\sim 0.6$ $0.3 \mathrm{eV}$ [the second-order exchange energy not present in the RPA is known to yield $\sim 0.6 \mathrm{eV}$ in the high-density $\left.\operatorname{limit}\left(r_{s} \rightarrow 0\right)\right]$, the quantity $\left\{\varepsilon_{x c}^{\text {unif }}[n(z)]-\varepsilon_{x c}^{\text {unif }}(\bar{n})\right\}$ entering Eq. (8) depends little on whether the RPA or the Perdew-Zunger parametrization is used, thereby yielding LDA xc surface energies that are comparable.

\section{SELF-CONSISTENT CALCULATIONS}

Due to the simple nature of the IBM one-electron wave functions, we have used this model as a proving ground for the calculation of both local (LDA) and non-local xc surface energies, and also for devising the extrapolation procedure dictated by Eq. (44). Nevertheless, the IBM yields unrealistic xc surface energies that are too small, especially at the lowest values of $r_{s}$ that we have explored, since at these high densities the infinite barrier constrains the electrons too close to the jellium background.

In this section, we apply Eq. (44) in the framework of a self-consistent microscopic model of the jellium slab. While in the IBM new subbands for the $z$ motion become occupied every time $d$ is a multiple of $\lambda_{F} / 2$ ( $x$ integer), for an arbitrary effective one-electron potential new subbands are expected to enter as $2 a / \lambda_{F}$ (not necessarily integer) is increased by approximately one. Accordingly, surface energies are still oscillatory functions of the slab thickness $a$, the period of the oscillations being approximately equal to $\lambda_{F} / 2$ and the amplitude decaying approximately linearly with $a$.

\section{A. LDA orbitals}

Self-consistent calculations of jellium surface energies of a semininfinite medium were carried out by Lang and Kohn ${ }^{3}$ with use of the LDA for exchange and correlation. They replaced the actual $v_{x c}[n](z)$ entering Eq. (7) by the LDA xc potential and computed the LDA sc surface energy from Eq. (8) using the Wigner form 36 for the correlation energy per particle of the uniform electron gas, $\varepsilon_{x c}^{\text {unif }}$. We have tested the approximation of Eq. (44) (with $\sigma$ replaced by our selfconsistent $\sigma_{x c}^{L D A}$ ), for slab widths as large as $\sim 10$ times the Fermi wavelength, and for $r_{s}=2$ we have found $\left[\sigma_{x c}^{L D A}\right]_{i w}=3256 \mathrm{erg} / \mathrm{cm}^{2}$, in agreement with the calculation reported by Lang and Kohn.41 Our self-consistent calculations of $\sigma_{x c}^{L D A} /\left[\sigma_{x c}^{L D A}\right]_{i w}$ are plotted in Fig. 7, together with the enveloping lines $\left[\sigma_{x c}^{L D A}\right]_{1} /\left[\sigma_{x c}^{L D A}\right]_{i w}$ and $\left[\sigma_{x c}^{L D A}\right]_{2} /\left[\sigma_{x c}^{L D A}\right]_{i w}$, as a function of the slab width (in units of $\lambda_{F} / 2$ ). Also represented in this figure are the extrapolated LDA xc surface energies (open circles), as obtained from Eq. (44) for those threshold slab widths $a_{n}$ for which the $n$th subband is first occupied. One clearly sees that self-consistent density profiles yield exactly the same oscillating behavior as obtained within the IBM, but now QSE are smaller and the extrapolation formula of Eq. (44) yields a result that for a slab width as little as $\sim 3.5$ times the Fermi wavelength $(n=7)$ js within $0.01 \%$ of the actual infinite-width calculation. 42

Now we focus on our RPA-based local and non-local calculations of the surface energy. As in the calculations of Lang and Kohn, we replace the actual $v_{x c}[n](z)$ entering Eq. (7) by the LDA xc potential 43 but with the Perdew-Zunger parametrization for the xc potential of the uniform electron gas. Local and non-local calculations of the xc surface energy are then carried out within one and the same density-response framework, i.e., by either introducing Eq. (15) into Eq. (8) or by introducing both Eqs. (15) and (18) into Eq. (5), with the use of the interacting RPA density-response function. As this framework is devoid of any ambiguities in the treatment of the many-body problem, it clearly serves as a measure of the actual error introduced by the LDA.

In Table II, we show the infinite-width limit of the various contributions to our RPA-based local (LDA) and non-local jellium surface energies, as obtained from Eq. (44) for slabs with $n=12\left(a \sim 5-6 \lambda_{F}\right)$.44 As in the case of IBM orbitals, self-consistent calculations show that the LDA systematically overestimates/underestimates the exchange/correlation surface energies, while the local xc surface energies differ little from their nonlocal counterparts. Table II shows that the percentage error between local and non-local exchange surface energies monotonically decreases, within the present model, from a minimum of $16 \%$ for the slow varying density at $r_{s}=2$ to a maximum of $95 \%$ for the more rapidly varying density at $r_{s}=6$. The percentage error between our local and non-local xc surface energies is found to vary from $1.8 \%$ at $r_{s}=2$ to $3.4 \%$ at $r_{s}=6$.

Self-consistent electron densities vary slowly as compared to the IBM, especially in the case of small values of $r_{s}$, as shown in Fig. 8. As a consequence, we find: (a) quantum-size effects are smaller [for slabs with 12 occupied levels the amplitude of the oscillations in both $\sigma_{x c}^{L D A}$ and $\sigma_{x c}$ ranges from $\sim 1 \%$ at $r_{s}=2$ to $\sim 5 \%$ at $\left.r_{s}=6\right]$; (b) all contributions to the surface energy are larger; and (c) the LDA is significantly better, over the entire metallic range of densities $\left(r_{s} \sim 2-6\right)$, than for the IBM electron-density profile.

The impact on the surface energy of the short-range correlations built into the many-body kernel of Eq. (13), not present in the RPA, was investigated in Ref. 45 within the ALDA. While introduction of the ALDA ker- 
nel slightly decreases the LDA xc surface energy [the ALDA-based LDA surface energies agree closely with the LDA quantum Monte Carlo calculations of Acioli and Ceperley 14], ALDA short-range corrections to the nonlocal RPA surface energy are found to be positive. As a result, the presence of the xc kernel enhances, within the ALDA, the discrepancy between local and non-local xc surface energies, but the error introduced by the LDA is still, within ALDA, of the order of $50 \%$ smaller than the error one would impute to the local apprgximation on the basis of the non-local FHNCA results, 12 particularly in the high-density region $\left(r_{s}=2\right)$.

\section{B. Hartree and exchange-only orbitals}

RPA-based local and non-local surface energies, as obtained with the use of Hartree orbitals, i.e., with the xc potential $v_{x c}[n]$ entering Eq. (而 set equal to zero, were reported in Ref. 45. Hartree orbitals are more delocalized than the more realistic LDA orbitals (with $v_{x c}[n](z)=0$ the work function is far too small and even negative at low values of $r_{s}$ ) and they yield a too slow varying electron-density profile, as shown in Fig. 8. This leads to surface energies that are too large, relative to those obtained with the use of LDA orbitals. We also find that the impact of xc on the one-electron states is much more pronounced than the impact of the short-range correlations built into the many-body kernel of Eq. (13).

For comparison, we have also computed kinetic, electrostatic and exchange surface energies with an LDA exchange-only effective one-electron potential $v_{x}[n](z)$, and have obtained the infinite-width extrapolated results shown in Table III. A comparison of the numbers presented in Tables II and III shows that the sum of kinetic, electrostatic and exchange surface energies is nearly insensitive to whether an LDA exchange-only or xc effective potential is used in the evaluation of the one-electron Kohn-Sham orbitals. Moreover, this sum (labeled $\sigma_{H F}$ in Table III) is expected to be very close to the $e x$ act Hartree-Fock surface energies. 46 Our exchange-only surface energy is for $r_{s}=2.07$ in excellent agreement with the Hartree-Fock surface energy of $-1273 \mathrm{erg} / \mathrm{cm}^{2}$ reported by Krotscheck and Kohn 12 Also, our exchangeonly surface energies fall for $r_{s}>3$ below the variational upper bound reported by Sahni and Ma 40

\section{SUMMARY AND CONCLUSIONS}

We have presented self-consistent-field calculations of the surface energy of a bounded electron gas. First of all, we have considered the infinite-barrier model of the surface. Within this model we have discussed the origin of quantum-size effects, and have devised a formula that accurately extrapolates our finite-slab calculations to the infinite-width limit. This formula may be useful to the case of more complex slab calculations involving the use of the band structure and/or quantum Monte Carlo simulations.

Subsequently, we have reported full self-consistent nonlocal calculations of the surface energy, by employing either LDA or the less realistic Hartree orbitals to construct the RPA density-response function. The unambiguous nature of the comparison of local versus non-local surface energies made possible by our self-consistent calculations leads us to the conclusion that the local-density approximation is accurate, within the RPA treatment of correlation.

Our non-local RPA surface energies, as computed with the use of realistic LDA orbitals, are in reasonable agreement with those obtained by Zhang, Langreth, and Perdew 15 using the non-local Langreth-Mehl xc functiona 48 and also with those obtained within the recently developed meta-generalized-gradient approximation (meta-GGA), 16 which makes use not only of the local density and its gradient but also of the orbital kineticenergy density. Moreover, a GGA 49 for the short-range correlation not present in the RPA has yielded a small and negative contribution to the surface energy, 17 thereby suggesting that the RPA may be a much better approximation for the changes in correlation energy upon surface formation than for the total correlation energy. A recent wave-vector interpolation as a long-range correction to the GGA, 18 which has predicted surface energies that are smaller but close to our non-local RPA results, has indicated that the LDA should be accurate even within an exact treatment of electron correlation. This is in contrast with non-local correlation surface energies reported in Refs. 12 and 14, which are significantly higher than our non-local RPA correlation energies and the local-density approximation.

\section{ACKNOWLEDGMENTS}

J.M.P. acknowledges partial support by the University of the Basque Country, the Basque Unibertsitate eta Ikerketa Saila, and the Spanish Ministerio de Educación y Cultura. A.G.E. acknowledges support from the National Science Foundation. The authors also acknowledge I. Sarria for his help in the preparation of the figures. ORNL is managed by Lockheed Martin Energy Research Corp. for the U.S. DOE under Contract No. DE-AC0596OR22464.

\section{APPENDIX A:}

Here we give an explicit expression for the xc surface energy of a jellium slab, in terms of the eigenvalues $\varepsilon_{l}$ and the Fourier coefficients of the eigenfunctions $\phi_{l}(z)$. It is also demonstrated that there is no contribution to 
the xc surface energy coming from large values of the momentum transfer parallel to the surface, so the xc energy per particle at point $z$ is devoid of divergences.

We first introduce the following double-cosine Fourier representations for the density-response function and the Dirac $\delta$ function:

$$
\chi\left(z, z^{\prime} ; q_{\|}, \omega\right)=\sum_{m=0}^{\infty} \sum_{n=0}^{\infty} \chi_{m n}\left(q_{\|}, \omega\right) \cos (m \pi \tilde{z}) \cos \left(n \pi \tilde{z}^{\prime}\right)
$$

and

$$
\delta\left(z-z^{\prime}\right)=\sum_{n=0}^{\infty} \frac{\mu_{m}}{d} \cos (n \pi \tilde{z}) \cos \left(n \pi \tilde{z}^{\prime}\right),
$$

where $d=a+2 z_{0}, \tilde{z}=z / d+1 / 2$, and $\mu_{m}$ is defined by the equation

$$
\mu_{m}= \begin{cases}1 & \text { for } m=0 \\ 2 & \text { for } m \geq 1\end{cases}
$$

The electron density can also be expressed in a cosine representation

$$
n(z)=\sum_{m=0}^{\infty} n_{m} \cos (m \pi \tilde{z})
$$

Eq. (6) yields

$$
n_{m}=\frac{\mu_{m}}{\pi d} \sum_{l=1}^{l_{M}}\left(E_{F}-\varepsilon_{l}\right) G_{l l}^{m}
$$

where

$$
G_{l l^{\prime}}^{m}=d \int_{0}^{d} d \tilde{z} \cos (m \pi \tilde{z}) \phi_{l}(\tilde{z}) \phi_{l^{\prime}}(\tilde{z}),
$$

or, in terms of the Fourier coefficients $b_{l s}$ of $\phi_{l}(z)$,

$$
G_{l l^{\prime}}^{m}=\frac{1}{2} \sum_{s=1}^{\infty} \sum_{s^{\prime}=1}^{\infty} b_{l s} b_{l^{\prime} s^{\prime}}\left(\delta_{m, s-s^{\prime}}+\delta_{m, s^{\prime}-s}-\delta_{m, s+s^{\prime}}\right) .
$$

Then, substituting Eq. (18) into Eq. (5), we obtain the following expression for the xc surface energy:

$$
\sigma_{x c}=\frac{1}{2} \int \frac{d \mathbf{q}_{\|}}{(2 \pi)^{2}} \sum_{m=0}^{\infty} \sum_{n=0}^{\infty} \alpha_{m n}\left(q_{\|}\right)-\bar{n} a \varepsilon_{x c}(\bar{n}),
$$

where

$$
\begin{aligned}
\alpha_{m n}\left(q_{\|}\right)= & v_{m n}\left(q_{\|}\right)\left[-\frac{\hbar}{\pi} \int_{0}^{1} d \lambda \int_{0}^{\infty} d \omega \chi_{m n}^{\lambda}\left(q_{\|}, i \omega\right)\right. \\
& \left.-\frac{\mu_{m} \mu_{n}}{\pi d^{2}} \sum_{l=1}^{l_{M}}\left(E_{F}-\varepsilon_{l}\right) \sum_{l^{\prime}=1}^{\infty} G_{l l^{\prime}}^{m} G_{l l^{\prime}}^{n}\right]
\end{aligned}
$$

and

$$
\begin{aligned}
v_{m n}\left(q_{\|}\right)= & \frac{2 \pi e^{2}}{q_{\|}^{2}+(m \pi / d)^{2}}\left[\frac{2 d}{\sqrt{\mu_{m} \mu_{n}}} \delta_{m n}\right. \\
& \left.-\left[1+(-1)^{m+n}\right] \frac{q_{\|}\left[1-(-1)^{m} \mathrm{e}^{-q_{\|} d}\right]}{\left(q_{\|}^{2}+(n \pi / d)^{2}\right.}\right] .
\end{aligned}
$$

As for the Fourier coefficients $\chi_{m n}^{\lambda}\left(q_{\|}, \omega\right)$, Eq. (12) yields

$$
\begin{aligned}
& \chi_{m n}^{\lambda}\left(q_{\|}, \omega\right)=\chi_{m n}^{0}\left(q_{\|}, \omega\right)+\sum_{m^{\prime}, n^{\prime}} \chi_{m m^{\prime}}^{0}\left(q_{\|}, \omega\right) \\
& \quad \times\left\{\lambda v_{m^{\prime} n^{\prime}}\left(q_{\|}\right)+f_{m^{\prime} n^{\prime}}^{x c, \lambda}[n]\left(q_{\|}, \omega\right)\right\} \chi_{n^{\prime} n}^{\lambda}\left(q_{\|}, \omega\right),
\end{aligned}
$$

where the Coulomb-interaction Fourier coefficients $v_{m n}\left(q_{\|}\right)$are given by Eq. (A10). An explicit expression for the coefficients $\chi_{m n}^{0}\left(q_{\|}, \omega\right)$ can be found in Ref. 28. $f_{m n}^{x c, \lambda}[n]\left(q_{\|}, \omega\right)$ are the Fourier coefficients of the xc kernel. In the RPA these coefficients are simply zero. In the ALDA,

$$
\begin{array}{rl}
f_{m n}^{x c, \lambda, A L D A}[n]\left(q_{\|}, \omega\right)=d \int_{0}^{d} & \left.d \tilde{z} \frac{d v_{x c}(n)}{d n}\right|_{n=n(z)} \\
& \times \cos (m \pi \tilde{z}) \cos (n \pi \tilde{z})
\end{array}
$$

If the Coulomb correlations entering Eq. (A11) are ignored altogether $(\lambda=0)$, the integrations over $\lambda$ and $\omega$ in Eq. (A9) can be performed analytically to find, after some algebra, the following result:

$$
\begin{aligned}
\alpha_{m n}\left(q_{\|}\right)= & v_{m n}\left(q_{\|}\right) \frac{\mu_{m} \mu_{n}}{\pi d^{2}} \sum_{l=1}^{l_{M}} \sum_{l^{\prime}=1}^{\infty} \\
& \times\left[F_{l l^{\prime}}\left(q_{\|}\right)-\left(E_{F}-\varepsilon_{l}\right)\right] G_{l l^{\prime}}^{m} G_{l l^{\prime}}^{n},
\end{aligned}
$$

where

$$
\begin{gathered}
F_{l l^{\prime}}\left(q_{\|}\right)=\varepsilon_{l} \operatorname{sgn}\left[a_{l l^{\prime}}\left(q_{\|}\right)\right]+\Theta\left[b_{l l^{\prime}}\left(q_{\|}\right)\right] \\
\times\left[\frac{a_{l l^{\prime}}\left(q_{\|}\right) \sqrt{b_{l l^{\prime}}\left(q_{\|}\right)}}{\pi q_{\|}^{2}}-\frac{2}{\pi} \varepsilon_{l} \arctan \frac{\sqrt{b_{l l^{\prime}}\left(q_{\|}\right)}}{a_{l l^{\prime}}}\right], \\
a_{l l^{\prime}}\left(q_{\|}\right)=\frac{\hbar}{2 m} q_{\|}^{2}-\frac{1}{\hbar}\left(\varepsilon_{l}-\varepsilon_{l^{\prime}}\right),
\end{gathered}
$$

and

$$
b_{l l^{\prime}}\left(q_{\|}\right)=2 q_{\|}^{2} \varepsilon_{l}-a_{l l^{\prime}}^{2}
$$

In the limit as $q_{\|} \rightarrow \infty$, we find

$$
\lim _{q_{\|} \rightarrow \infty} F_{l l^{\prime}}\left(q_{\|}\right)=\left(E_{F}-\varepsilon_{l}\right) .
$$

As in this limit the interacting density-response function approaches its non-interacting counterpart, this result shows, explicitly, that there is no contribution to the 
xc surface energy coming from high values of the parallel component of the momentum transfer, and that the singular term $\delta\left(z-z^{\prime}\right)$ in Eq. (18) gives no divergences.

${ }^{1}$ P. Hohenberg and W. Kohn, Phys. Rev. 136, B864 (1964).

${ }^{2}$ W. Kohn and L. Sham, Phys. Rev. 140, A1133 (1965).

${ }^{3}$ N. D. Lang and W. Kohn, Phys. Rev. B 1, 4555 (1970).

${ }^{4}$ N. D. Lang, Solid State Phys. 28, 225 (1973).

${ }^{5}$ J. Harris and R. O. Jones, J. Phys. F 4, 1170 (1974).

${ }^{6}$ N. D. Lang and L. J. Sham, Solid State Commun. 17, 581 (1975).

${ }^{7}$ M. Rasolt and D. J. W. Geldart, Phys. Rev. Lett. 35, 1234 (1975); J. H. Rose, Jr., H. B. Shore, D. J. W. Geldart, and M. Rasolt, Solid State Commun. 19, 619 (1976).

${ }^{8}$ E. Wikborg and J. E. Inglesfield, Solid State Commun. 16, 335 (1975).

${ }^{9}$ D. C. Langreth and J. P. Perdew, Solid State Commun. 17, 1425 (1975); Phys. Rev. B 15, 2884 (1977).

10 J. P. Perdew, D. C. Langreth, and V. Sahni, Phys. Rev. Lett. 38, 1030 (1977)

${ }^{11}$ D. C. Langreth and J. P. Perdew, Solid State Commun. 31, 567 (1979); Phys. Rev. B 21, 5469 (1980).

12 E. Krotscheck, W. Kohn, and G.-X Qian, Phys. Rev. B 32, 5693 (1985); E. Krotscheck and W. Kohn, Phys. Rev. Lett. 57, 862 (1986).

${ }^{13}$ X.-P. Li, R. J. Needs, R. M. Martin, and D. M. Ceperley, Phys. Rev. B 45, 6124 (1992).

${ }^{14}$ P. H. Acioli and D. M. Ceperley, Phys. Rev. B 54, 17199 (1996).

15 Z. Y. Zhang, D. C. Langreth, and J. P. Perdew, Phys. Rev. B 41, 5674 (1990).

16 J. P. Perdew, S. Kurth, A. Zupan, and P. Blaha, Phys. Rev. Lett. 82, 2544 (1999).

17 S. Kurth and J. P. Perdew, Phys. Rev. B 59, 10461 (1999).

${ }^{18}$ Z. Yan, J. P. Perdew, S. Kurth, C. Fiolhais, and L. Almeida, Phys. Rev. B 61, 2595 (2000).

${ }^{19}$ G. D. Mahan, Many-Particle Physics, 2nd ed. (Plenum, New York, 1990).

${ }^{20}$ F. K. Schulte, Surf. Sci. 55, 427 (1976).

${ }^{21}$ P. J. Feibelman, Phys. Rev. B 27, 1991 (1983); P. J. Feibelman and D. R. Hamann, Phys. Rev. B 29, 6463 (1984).

${ }^{22}$ H. B. Callen and T. R. Welton, Phys. Rev. 83, 34 (1951).

${ }^{23}$ E. K. U. Gross, J. F. Dobson, and M. Petersilka, in Density Functional Theory II, Vol. 181 of Topics in Current Chemistry, edited by R. F. Nalewajski (Springer, Berlin, 1996), p. 81.

${ }^{24}$ E. Runge and E. K. U. Gross, Phys. Rev. Lett. 52, 997 (1984).

${ }^{25}$ This DFT-based RPA differs from the less realistic actual RPA, which is constructed from the $\chi^{0}\left(\mathbf{r}, \mathbf{r}^{\prime} ; \omega\right)$ densityresponse function of independent electrons with a selfconsistent Hartree hamiltonian.

${ }^{26}$ A. Zangwill and P. Soven, Phys. Rev. A 21, 1561 (1980).

27 J. Lindhard, K. Dan. Vidensk. Selsk. Mat.-Fys. Medd. 28 (8), 1 (1954).
${ }^{28}$ A. G. Eguiluz, Phys. Rev. Lett. 51, 1907 (1983); Phys. Rev. B 31, 3303 (1985).

${ }^{29}$ In the non-selfconsistent infinite-barrier model (IBM), $z_{0}$ is chosen so as to ensure charge neutrality. For a selfconsistent description of the density-response function, $z_{0}$ is chosen sufficiently large for the physical results to be insensitive to the precise value employed.

30 J. Bardeen, Phys. Rev. 49, 653 (1936).

${ }^{31}$ D. M. News, Phys. Rev. 1, 3304 (1971).

32 A. Griffin and J. Harris, Can. J. Phys. 54, 1396 (1976).

${ }^{33}$ E. Wikborg and J. E. Inglesfield, Phys. Scr. 15, 37 (1977).

${ }^{34}$ M. Abramowitz and I. A. Stegun, Handbook of Mathematical Functions (Dover, New York, 1965).

${ }^{35}$ Since the so-called electron-density parameter $r_{s}$, defined by the relation $1 / \bar{n}=(4 / 3) \pi r_{s}^{3}$, is usually given in units of the Bohr radius, $a_{0}$, we note that $e^{2} / a_{0}^{3}=1.5569 \times$ $10^{6} \mathrm{erg} / \mathrm{cm}^{2}$.

${ }^{36}$ E. P. Wigner, Phys. Rev. 46, 1002 (1934); Trans. Faraday Soc. 34, 678 (1938); see, also, D. Pines, Elementary Excitations in Solids (Addison Wesley, New York, 1963).

37 J. P. Perdew and A. Zunger, Phys. Rev. B 23, 5048 (1981).

${ }^{38}$ D. M. Ceperley and B. J. Alder, Phys. Rev. Lett. 45, 1196 (1980).

${ }^{39}$ We estimate that the numerical error introduced by our slab calculations corresponds to one unit in the last digit of all the entries in Tables I, II, and III.

${ }^{40}$ Small differences between our calculated xc surface energies and those reported by Wikborg and Inglesfield 8 are due to the fact that contributions from $q_{\|}>2 q_{F}$ to the integral of Eq. (18) were omitted by these authors.

${ }^{41}$ For slabs widths of $\sim 5-6$ times the Fermi wavelength $(n=12)$, Eq. (44) has been found to reproduce all kinetic, electrostatic, and LDA xc surface energies reported in Ref. 3 for semi-infinite media.

42 Due to the subtle cancellations that exist among the various contributions to the surface energy, the introduction of small errors in the kinetic, electrostatic, and xc terms may result in larger uncertainties of the total surface energy.

43 The use of the LDA xc potential and, therefore, LDA orbitals, should not be confused with the use of the LDA to replace $\varepsilon_{x c}[n](z)$ in Eq. (5) by the xc energy per particle of a uniform electron gas of density $n(z), \varepsilon_{x c}^{\text {unif }}[n(z)]$.

${ }^{44}$ Differences between the RPA-based LDA surface nergies reported in Table II and those of Lang and Kohn most entirely due to the use by these authors of the Wigner formula for the xc energy entering Eq. (8), instead of the RPA xc energy that we obtain from Eq. 15). Differences also appear as a result of these authors evaluating the xc potential entering Eq. (7) by using the Wigner parametrization; however, we have found that the various contributions to both local and nonlocal surface energies are rather insensitive to the actual choice of the xc potential of the uniform electron gas, and that the total surface energy is even less sensitive to this choice. Furthermore, although a fully self-consistent non-local treatment of surface energies would require a nonlocal calculation of the xc potential $v_{x c}[n](z)$, the error introduced by the use of LDA orbitals is expected to be negligible.

${ }^{45}$ J. M. Pitarke and A. G. Eguiluz, Phys. Rev. B 57, 6329 (1998). 
${ }^{46}$ Differences between the non-local Hartree-Fock surface energies presented in Table III and exact Hartree-Fock surface energies are due to our use of LDA exchange-only orbitals, which may differ from the actual eigenfunctions of the nonlocal Hartree-Fock hamiltonian.

${ }^{47}$ V. Sahni and C. Q. Ma, Phys. Rev. B 22, 5987 (1980).

${ }^{48}$ D. C. Langreth and M. J. Mehl, Phys. Rev. Lett. 47, 446 (1981).

49 J. P. Perdew, K. Burke, and M. Ernzerhof, Phys. Rev. Lett. 77, 3865 (1996).

TABLE I. Non-local RPA correlation $\left(\sigma_{c}\right)$ and exchange-correlation $\left(\sigma_{x c}\right)$ surface energies obtained in the infinite-barrier model of the surface, and their local counterparts. Also shown are the LDA xc surface energies $\left(\sigma_{x c}^{L D A^{\prime}}\right)$ obtained by using the Perdew-Zunger interpolation formula for the correlation energy of a uniform electron gas. Units are $\mathrm{erg} / \mathrm{cm}^{2}$.

\begin{tabular}{lcccccc}
\hline \hline$r_{s}$ & $\sigma_{c}$ & $\sigma_{c}^{L D A}$ & $\sigma_{c}^{L D A^{\prime}}$ & $\sigma_{x c}$ & $\sigma_{x c}^{L D A}$ & $\sigma_{x c}^{L D A^{\prime}}$ \\
\hline 2.0 & 679 & 146 & 127 & 1471 & 1376 & 1357 \\
2.07 & 617 & 136 & 118 & 1331 & 1245 & 1227 \\
3.0 & 219 & 60 & 52 & 454 & 424 & 416 \\
4.0 & 99 & 31 & 26 & 198 & 185 & 180 \\
5.0 & 53 & 18 & 15 & 104 & 97 & 94 \\
6.0 & 33 & 12 & 10 & 62 & 58 & 56 \\
\hline \hline
\end{tabular}

TABLE II. Kinetic $\left(\sigma_{s}\right)$, electrostatic $\left(\sigma_{e s}\right)$, exchange $\left(\sigma_{x}\right)$, non-local RPA xc $\left(\sigma_{x c}\right)$, and total $(\sigma)$ surface energies and their local counterparts, as obtained with the use of Perdew-Zunger parametrized LDA orbitals. Units are $\mathrm{erg} / \mathrm{cm}^{2}$.

\begin{tabular}{lcccccccc}
\hline \hline$r_{s}$ & $\sigma_{s}$ & $\sigma_{e s}$ & $\sigma_{x}$ & $\sigma_{x}^{L D A}$ & $\sigma_{x c}$ & $\sigma_{x c}^{L D A}$ & $\sigma$ & $\sigma^{L D A}$ \\
\hline 2.00 & -5495 & 1276 & 2624 & 3037 & 3467 & 3405 & -752 & -814 \\
2.07 & -4643 & 1072 & 2296 & 2674 & 3064 & 3007 & -507 & -564 \\
2.30 & -2750 & 627 & 1521 & 1809 & 2098 & 2054 & -25 & -69 \\
2.66 & -1316 & 299 & 854 & 1051 & 1240 & 1211 & 223 & 194 \\
3.00 & -703 & 164 & 526 & 669 & 801 & 781 & 262 & 242 \\
3.28 & -435 & 105 & 364 & 477 & 579 & 563 & 249 & 233 \\
4.00 & -139 & 42 & 157 & 222 & 278 & 269 & 181 & 172 \\
5.00 & -30 & 17 & 57 & 92 & 119 & 115 & 106 & 102 \\
6.00 & -3 & 9 & 22 & 43 & 58 & 56 & 64 & 62 \\
\hline \hline
\end{tabular}

TABLE III. As in Table II, but with use of exchange-only LDA orbitals.

\begin{tabular}{lcccccc}
\hline \hline$r_{s}$ & $\sigma_{s}$ & $\sigma_{e s}$ & $\sigma_{x}$ & $\sigma_{x}^{L D A}$ & $\sigma_{H F}$ & $\sigma_{H F}^{L D A}$ \\
\hline 2.00 & -5707 & 1390 & 2726 & 3131 & -1591 & -1186 \\
2.07 & -4832 & 1172 & 2390 & 2767 & -1270 & -899 \\
3.00 & -770 & 189 & 568 & 707 & -13 & 126 \\
4.00 & -169 & 49 & 180 & 243 & 60 & 123 \\
5.00 & -46 & 19 & 71 & 105 & 44 & 78 \\
6.00 & -13 & 9 & 32 & 52 & 28 & 48 \\
\hline \hline
\end{tabular}

FIG. 1. The solid line represents the normalized average electron density $\rho_{0}$, as obtained from Eq. (26), versus the parameter $x=2 d / \lambda_{F}$. Dashed lines represent $\left[\rho_{0}\right]_{1}$ and $\left[\rho_{0}\right]_{2}$, as obtained from Eqs. (28) and (29). The dotted line represents the infinite-width limit of Eq. (24).

FIG. 2. As in Fig. 1, for the normalized electron density at the center of the slab, obtained from Eqs. (32), (33), and (34). The dotted line represents the infinite-width limit, $[\rho(0)]_{i w}=1$.

FIG. 3. As in Fig. 1, for $z_{0} /\left[z_{0}\right]_{i w}$ obtained from Eqs. (27), (35), and (36).

FIG. 4. As in Fig. 1, for $\sigma_{s} /\left[\sigma_{s}\right]_{i w}$ obtained from Eqs. (37), (39), and (40).

FIG. 5. The solid line represents the normalized LDA exchange surface energy, $\sigma_{x}^{L D A} /\left[\sigma_{x}^{L D A}\right]_{i w}$, as obtained from Eq. (17) for the IBM electron density of a finite slab, versus the parameter $x=2 d / \lambda_{F}$. Dashed lines represent the envelopes $\left[\sigma_{x}^{L D A}\right]_{1} /\left[\sigma_{x}^{L D A}\right]_{i w}$ and $\left[\sigma_{x}^{L D A}\right]_{2}^{ \pm} /\left[\sigma_{x}^{L D A}\right]_{i w}$. The extrapolated normalized LDA surface energies, as obtained from Eq. (44) with $\sigma$ replaced by $\sigma_{x}^{L D A}$, are represented by open circles.

FIG. 6.6 The solid lines represent the envelopes $\left[\sigma_{x}\right]_{1} /\left[\sigma_{x}\right]_{i w}$ and $\left[\sigma_{x}^{L D A}\right]_{2}^{ \pm} /\left[\sigma_{x}\right]_{i w}$ of the normalized exact exchange surface energies, which have been obtained, within the IBM, by introducing Eqs. (15) and (18) into Eq. (5) with $\chi^{0}(q, \omega)$ and $\chi^{0}\left(z, z^{\prime} ; q_{\|}, \omega\right)$ instead of $\chi^{\lambda}(q, \omega)$ and $\chi^{\lambda}\left(z, z^{\prime} ; q_{\|}, \omega\right)$. The corresponding LDA enveloping lines (also shown in Fig. 5) are represented by dashed lines. Open squares and circles represent extrapolated exact (squares) and LDA (circles) exchange surface energies, as obtained from the procedure dictated by Eq. (44).

FIG. 7. The solid line represents the normalized LDA xc surface energy, $\sigma_{x c}^{L D A} /\left[\sigma_{x c}^{L D A}\right]_{i w}$, as obtained from Eq. (8) for the Lang-Kohn electron-density profile of a finite slab, versus the slab width (in units of $\lambda_{F} / 2$ ). Dashed lines represent the envelopes $\left[\sigma_{x}^{L D A}\right]_{1} /\left[\sigma_{x}^{L D A}\right]_{i w}$ and $\left[\sigma_{x}^{L D A}\right]_{2}^{ \pm} /\left[\sigma_{x}^{L D A}\right]_{i w}$. The extrapolated normalized LDA surface energies, as obtained from Eq. (44) with $\sigma$ replaced by $\sigma_{x c}^{L D A}$, are represented by open circles. 
FIG. 8. The normalized electron-density profile of a jellium surface with $r_{s}=2.07$, as obtained with use of Perdew-Zunger parametrized LDA orbitals (solid line), Hartree orbitals (dotted line), and within the IBM (dashed line). We note that the normalized IBM electron-density profile, with the $z$ coordinate measured in units of the Fermi wavelength, is independent of $r_{s}$. In this figure, the jellium edge has been taken at $z=0$. 
Figure 1

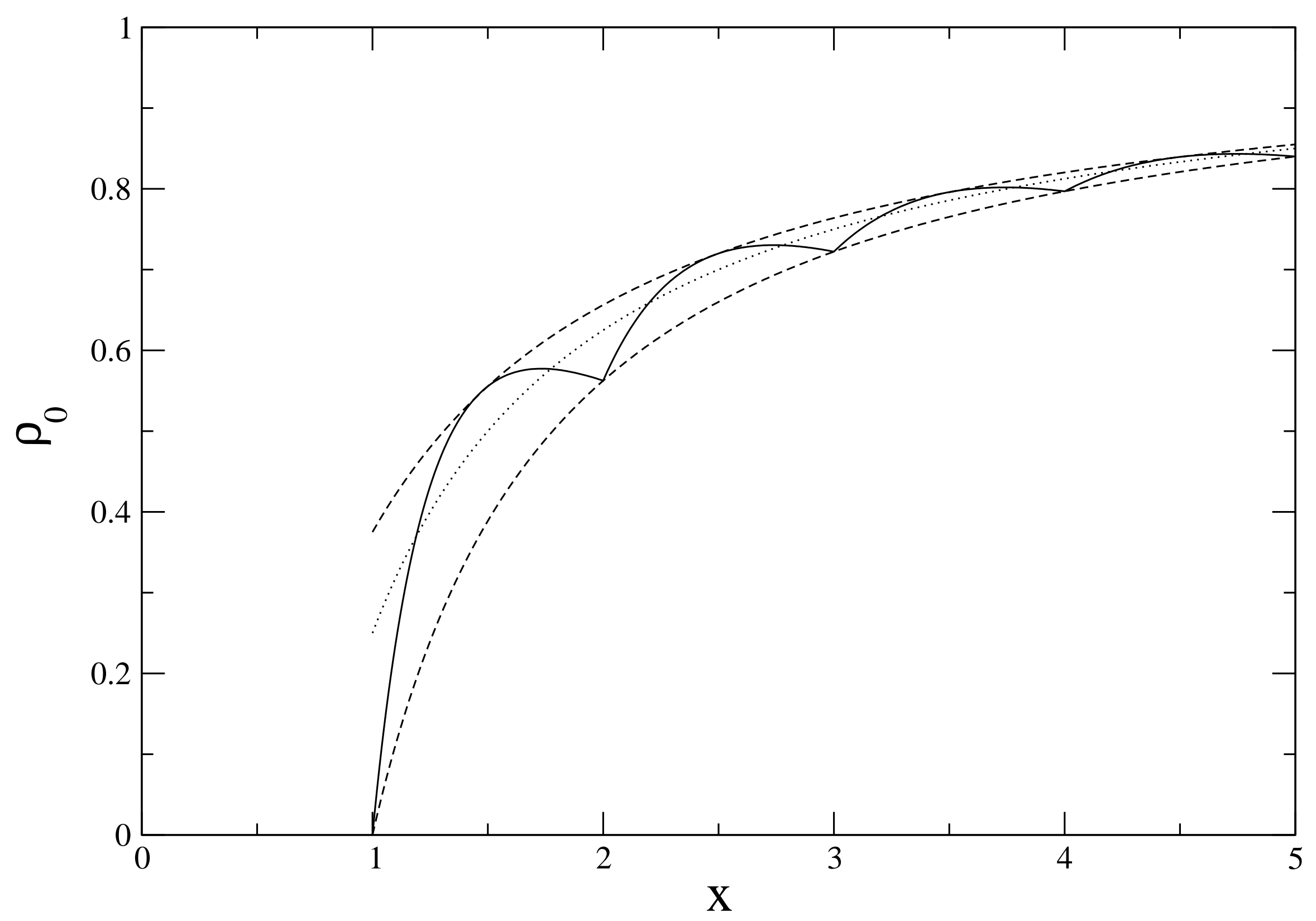


Figure 2

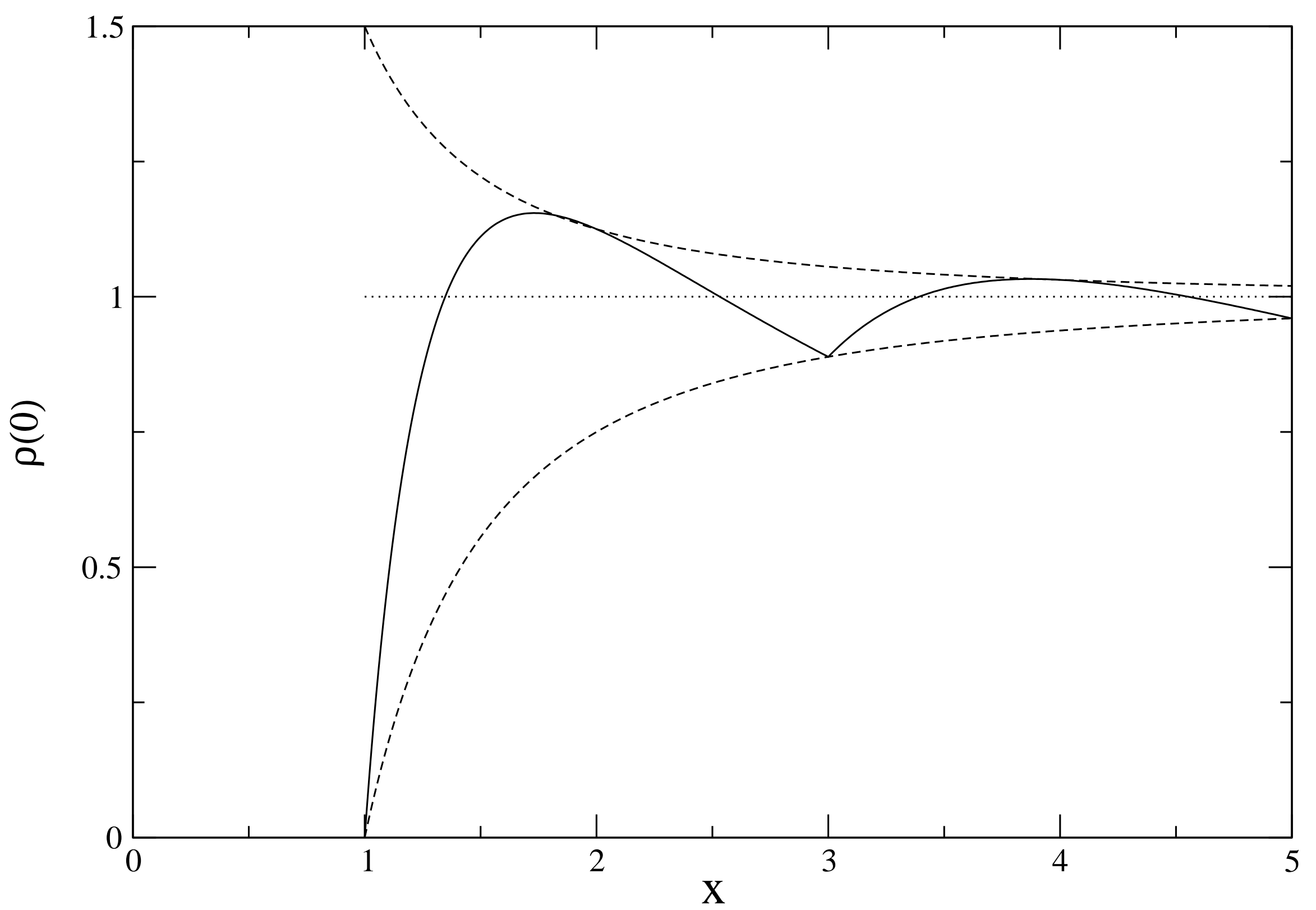


Figure 3

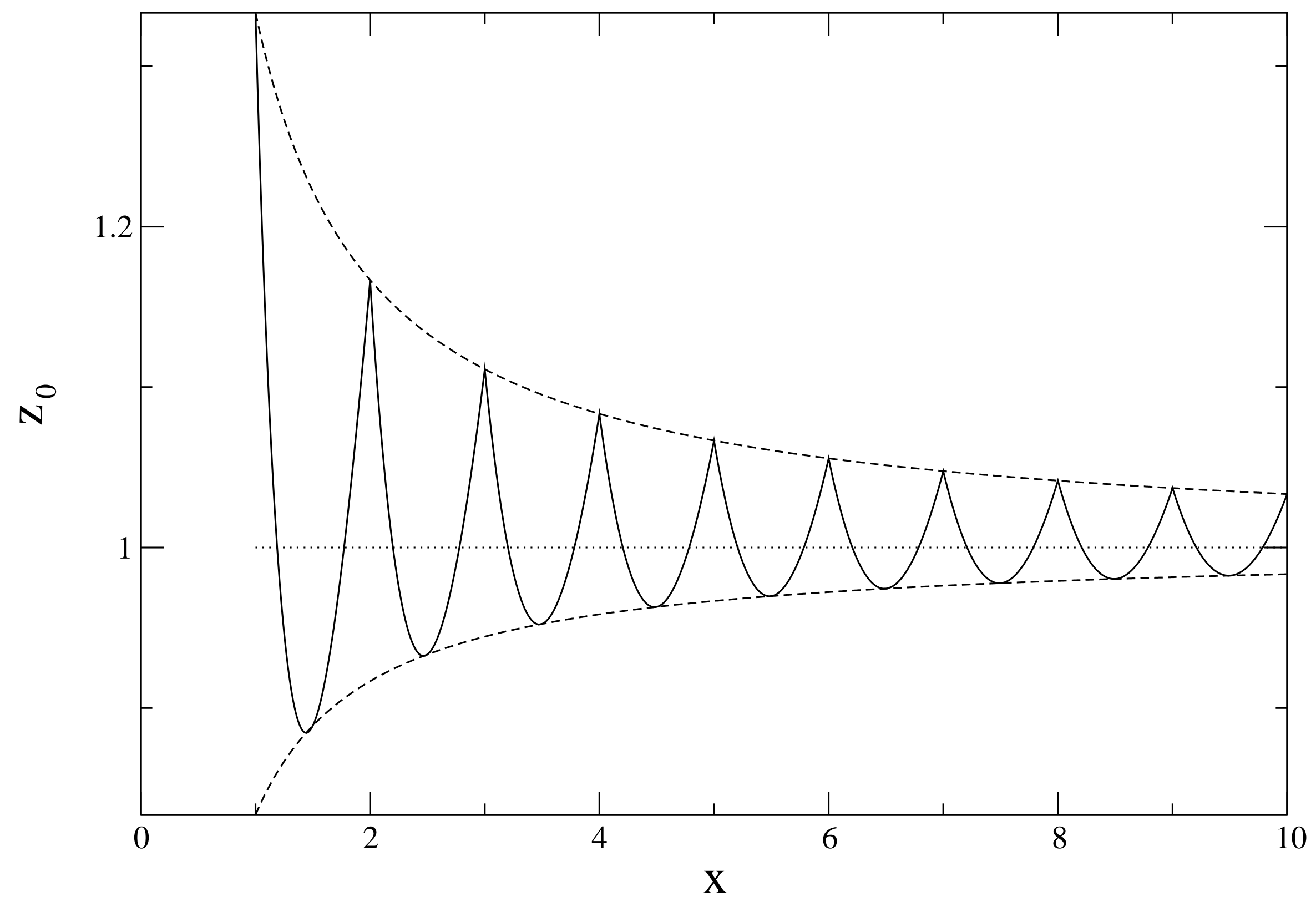


Figure 4

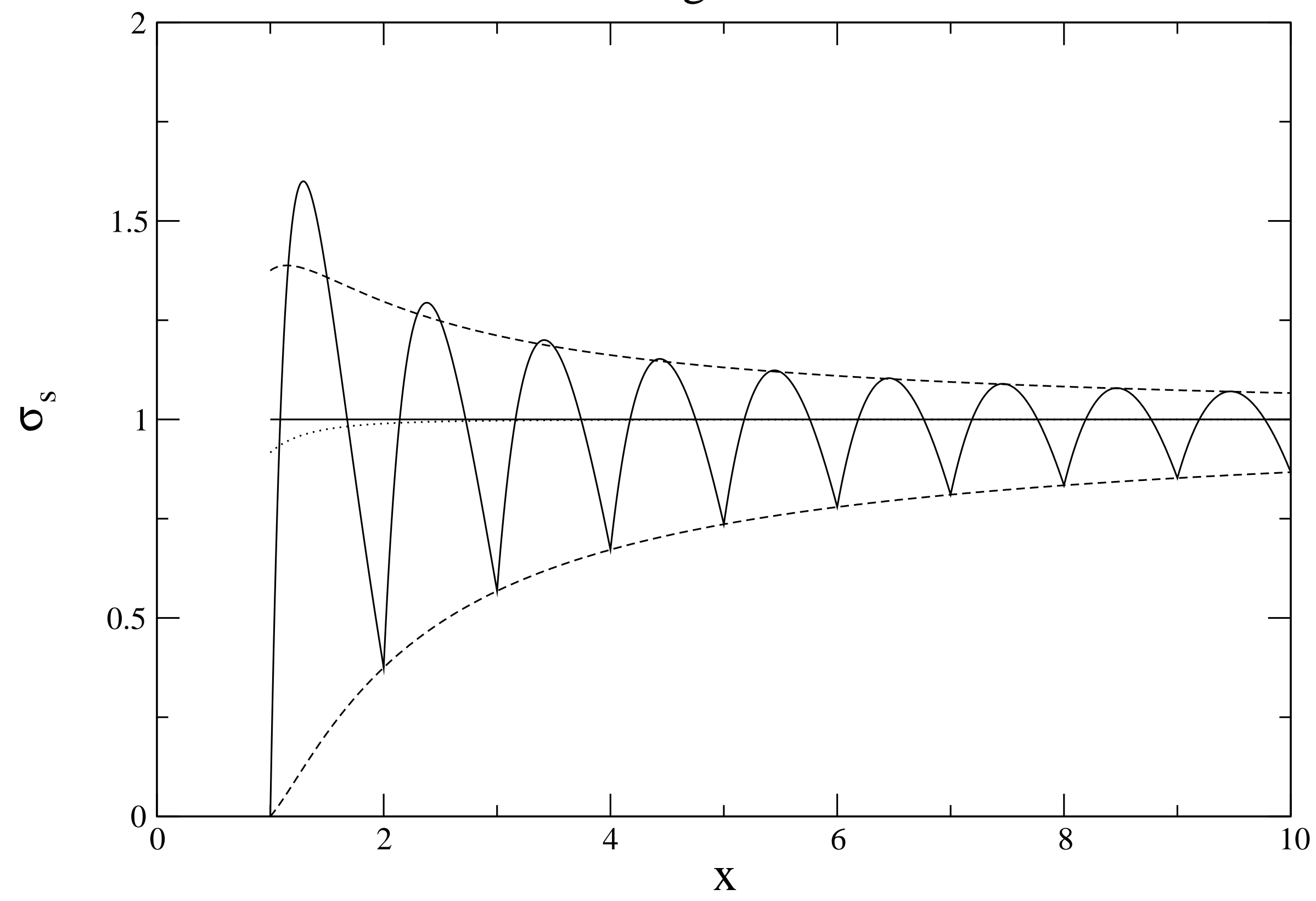


Figure 5

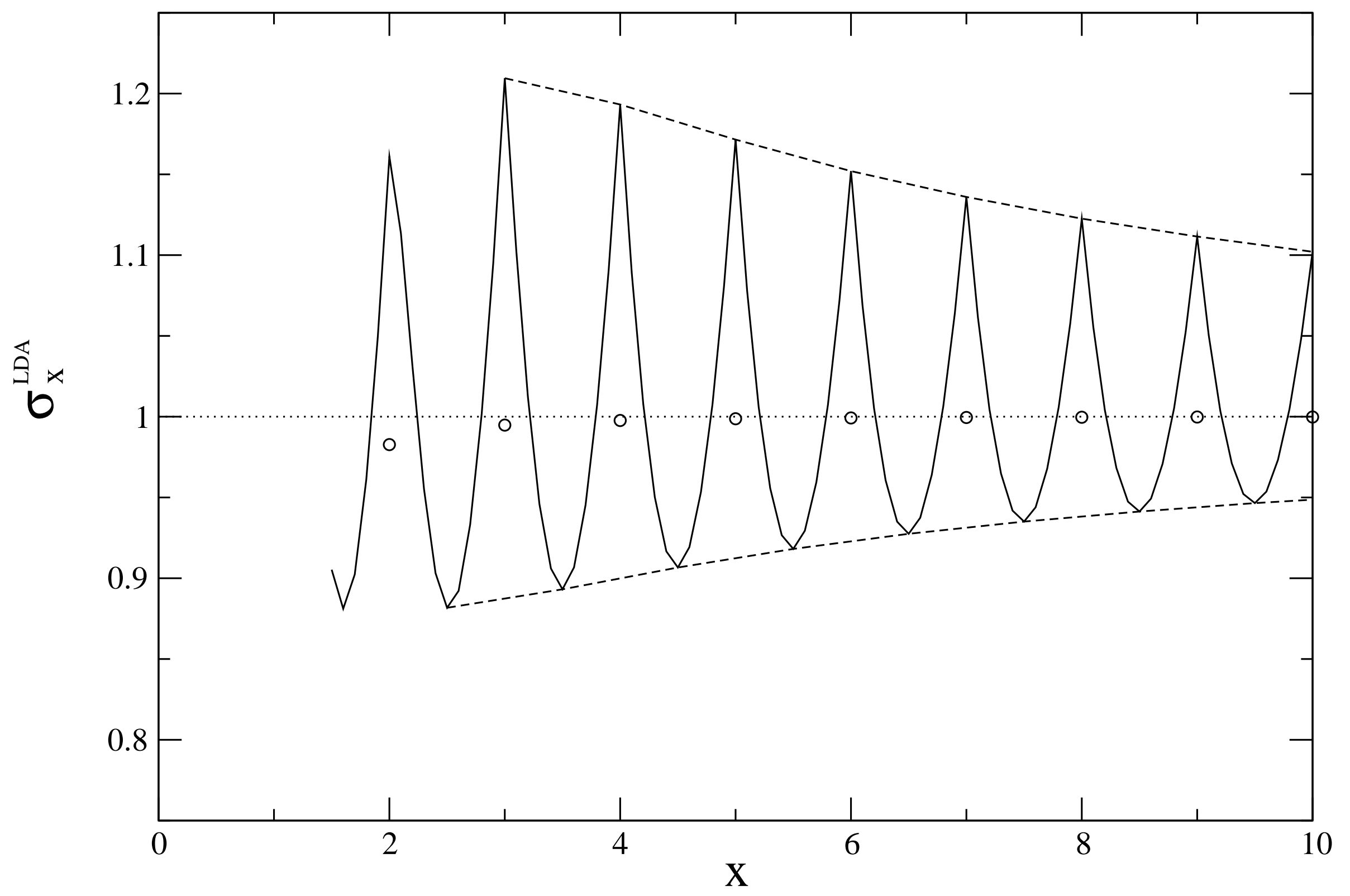


Figure 6

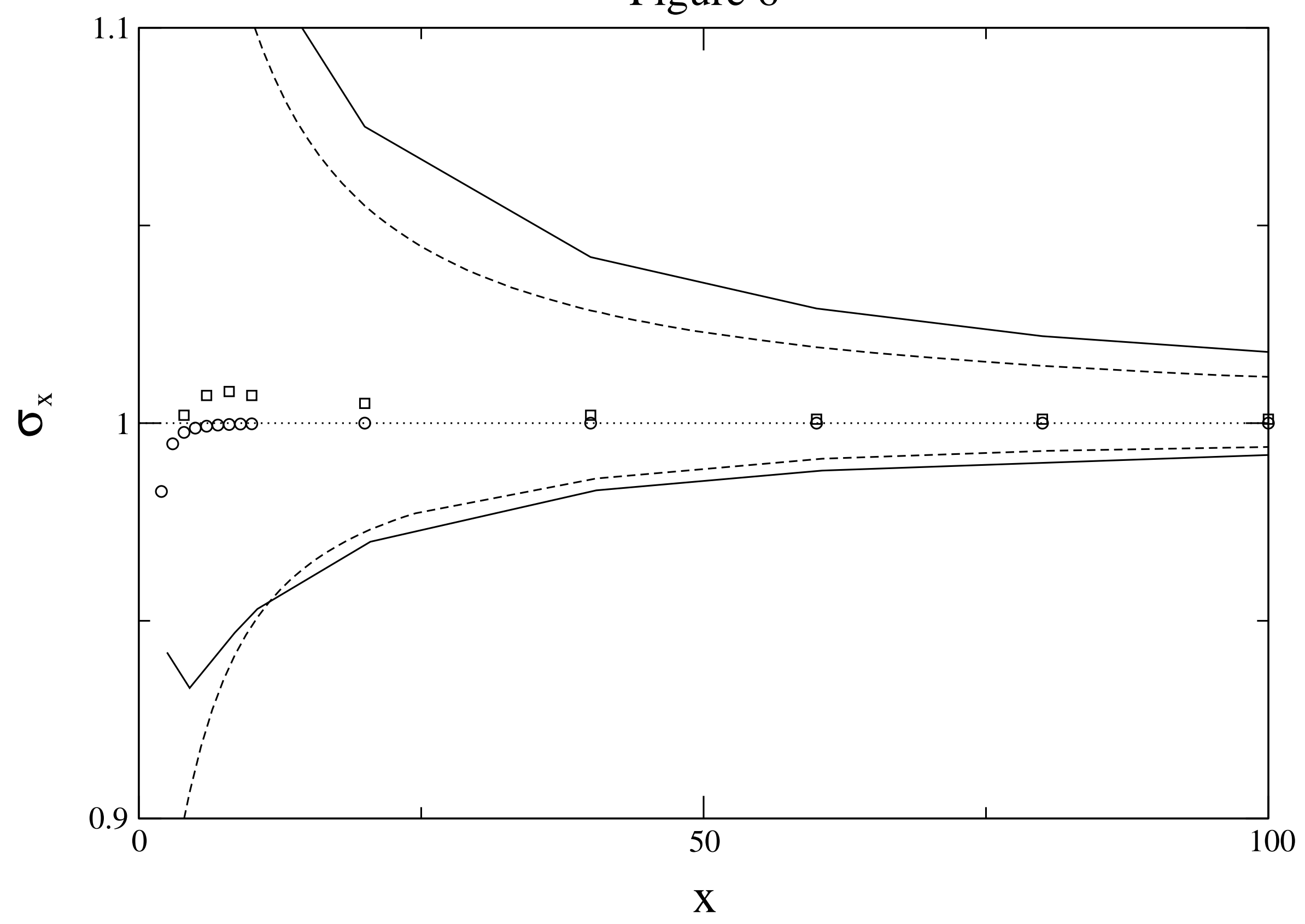


Figure 7

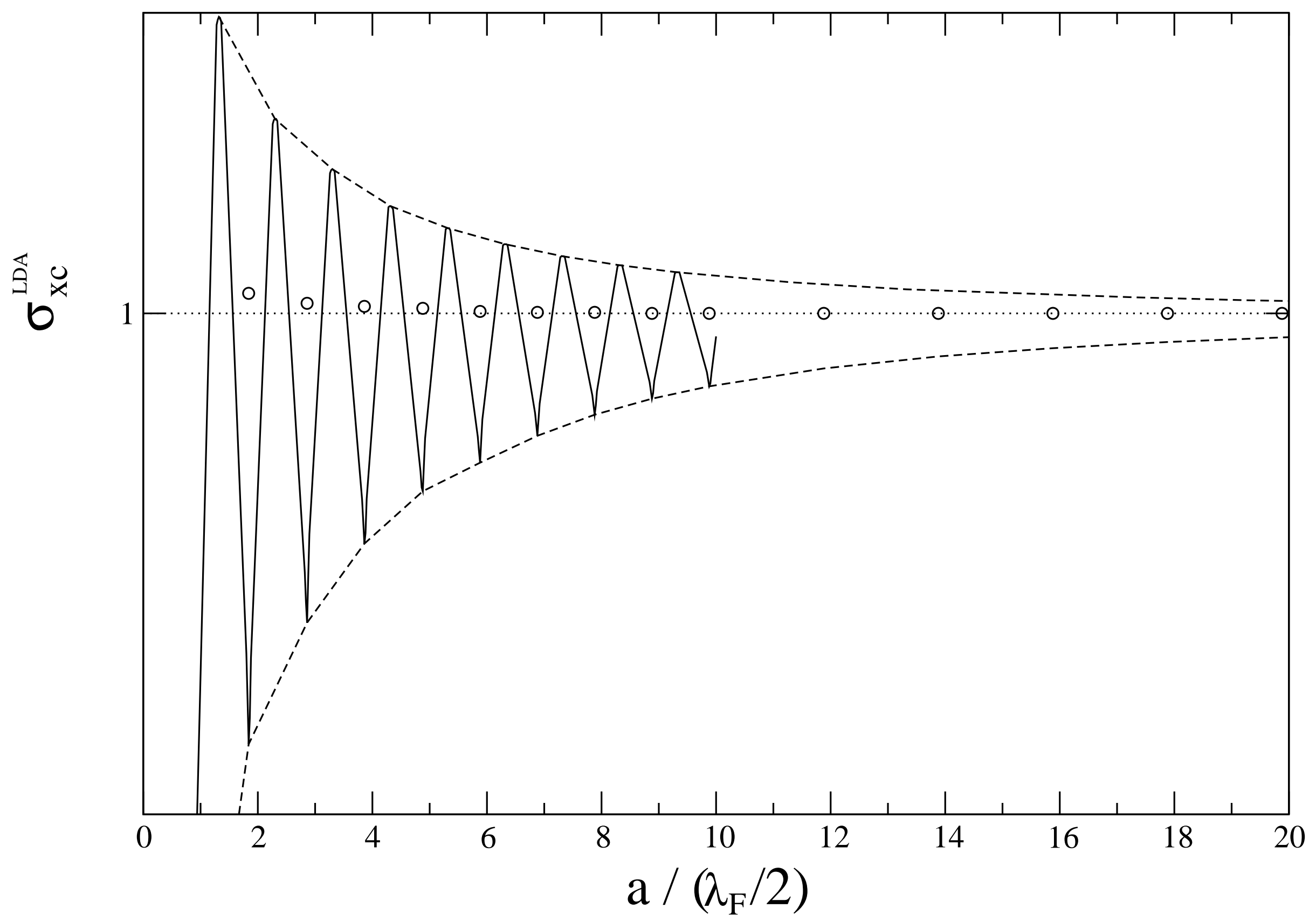


Figure 8

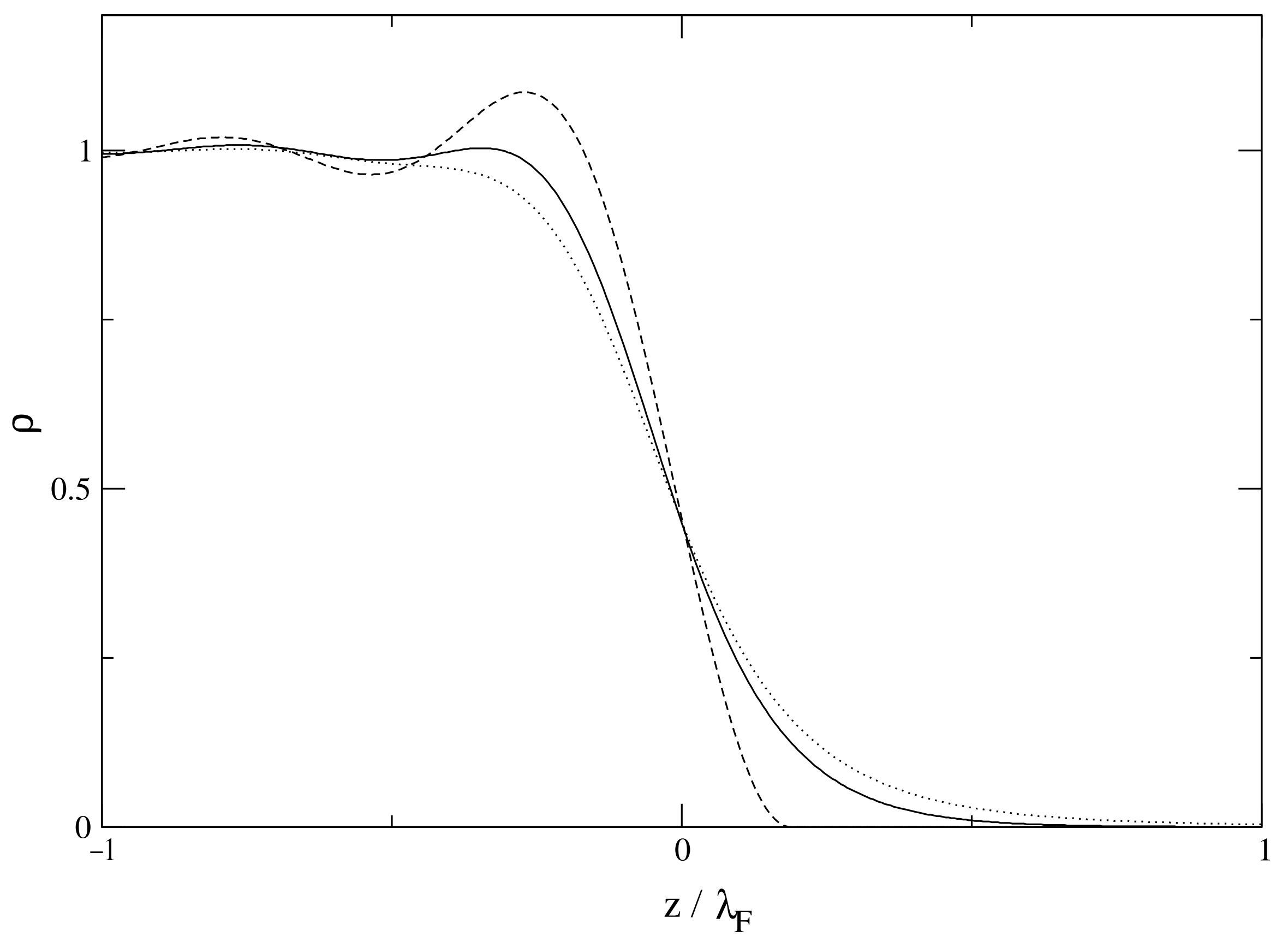

